\title{
The Research into a Two-directional matrix-valued Quarternary Wave Wraps with multi-scale and Applications
}

\author{
Shiheng Wang, Chunyi Jiao
}

Department of Fundamentals, Nanyang Vocational College of Agriculture, Nanyang 473000, China sxxa11xauat@126.com

\begin{abstract}
Keywords: Wavelet analysis, Vector scaling function, four-dimensional, vector-valued wavelet pac -kets, frame packets, vector subdivision scheme, biorthogonality formulas

Abstract. In this paper, we introduce a class of vector-valued four-dimensional wavelet packets according to a dilation matrix, which are generalizations of univariate wavelet packets. The defini -tion of biorthogonal vector four-dimensional wavelet packets is provided and their biorthogonality quality is researched by means of time-frequency analysis method, vector subdivision scheme and functional analysis method. Three biorthogonality formulas regarding the wavelet packets are es -tablished. Finally, it is shown how to draw new Riesz bases of space $L^{2}\left(R^{4}\right)^{n}$ from these wavelet packets. The sufficient condition for the existence of four-dimensional wavelet packets is estab -lished based on the multiresolution analysis method.
\end{abstract}

\section{Introduction}

Mechanical engineering is a discipline of engineering that applies the principles of physics and materials science for analysis, design, manufacturing, and maintenance of mechanical systems. It is the branch of engineering that involves the production and usage of heat and mechanical power for the design, production, and operation of machines and tools. It is one of the oldest and broadest engi -neering disciplines. Chen [1] introduced the notion of orthogonal vector-valued wavelets [2], Every frame(or Bessel sequence) determi-nes an analysis operator, the range of which is important for a lumber of applications.prefiltering is usually required for discrete multiwavelet transforms [3] but not necessary for discrete vector-valued transforms. Wavelet packets, owing to their nice characterristics, have been widely applied to signal processing [4], code theory[5], image compression[6], solving integral equation and so on. Coifman and Meyer firstly introduced the notion of univariate orthogonal wavelet packets. Yang [7] constructed a-scale orthogonal multiwavelet packets which were more flexible in applications. It is known that the majority of information is multi-dimensional information. Shen [8] introduced multivariate orthogonal wavelets which may be used in a wider field. Thus, it is necessary to generalize the concept of multivariate wavelet packets to the case of multivariate vector-valued wavelets.

\section{Notations and preliminaries on vector-valued function space}

We start from some notations. Set $Z_{+}=\{0\} \bigcup N, s, n, v \in N$ and $s, n, v \geq 2, Z^{4}=\left\{\left(z_{1}, z_{2}, z_{3}, z_{4}\right)\right.$ : $\left.z_{r} \in Z, r=1,2,3,4\right\}, Z_{+}^{4}=\left\{\left\{\left(z_{1}, z_{2}, z_{3}, z_{4}\right): z_{r} \in z_{+}, r=1,2,3,4\right\}\right.$. Assume that $S$ is an $4 \times 4$ matrix whose all entries are integers and all eigenvalues is large than one in modulus. The absolute value of the 
determinant of matrix $S$ is denoted by, i.e., $|\operatorname{det}(S)|=a$. Order $S^{T}$ stands for the transpose of matrix, and $S^{-T}$ is the inverse of the transpose of matrix $S$. For $V, V_{1}, V_{2} \subset R^{4}$, denoting by $S V=\{S x: x \in V\}$ $V_{1}+V_{2}=\left\{x_{1}+x_{2}: x_{1} \in V_{1}, x_{2} \in V_{2}\right\}, V_{1}-V_{2}=\left\{x_{1}=\left\{x_{1}-x_{2}: x_{1} \in V_{1}, x_{2} \in V_{2}\right\}\right.$. There are $a$ elements $\mu_{0}, \mu_{1}$, $\cdots, \mu_{a-1}$ in $Z_{+}^{4}$ by the finite group theory such that $Z^{s}=\bigcup_{\mu \in \Gamma_{0}}\left(\mu+S Z^{4}\right) ;\left(\mu_{1}+S Z^{4}\right) \cap\left(\mu_{2}+S Z^{4}\right)=\emptyset$, where $\Gamma_{0}=\left\{\mu_{0}, \mu_{1}, \cdots, \mu_{a-1}\right\}$ denotes the set of all different representative elements in the quotient group $Z^{4} /\left(S Z^{4}\right)$ and $\mu_{1}, \mu_{2}$ denote two arbitrary distinct elements in $\Gamma_{0}$. Set $\mu_{0}=\underline{0}$, where $\underline{0}$ is the null of $Z_{+}^{4}$. Let $\Gamma=\Gamma_{0}-\{\underline{0}\}$ and $\Gamma, \Gamma_{0}$ be two index sets. By $L^{2}\left(R^{4}\right)^{n}$, we denote the set of all vector functions $\Phi(x)$, i.e., $L^{2}\left(R^{4}\right)^{n}:=\left\{\lambda(x)=\left(\lambda_{1}(x), \lambda_{2}(x), \cdots \lambda_{n}(x)\right)^{T}: \lambda_{1}(x), \lambda_{2}(x), \cdots \lambda_{n}(x) \in L^{2}\left(R^{4}\right)\right\}$, where $T$ means the transpose of a vector. Video images and digital films are examples of vector functions where $\lambda_{l}(x)$ in the above $\lambda(x)$ denotes the pixel on the $l$ th column at the point $x$. For $\lambda(x) \in L^{2}\left(R^{4}\right)^{n}, \quad\|\lambda\|_{2}$ denotes the norm of vector function $\lambda(x)$, i.e., $\|\lambda\|_{2}:=\left(\sum_{l=1}^{v} \int_{R^{4}}\left|\lambda_{l}(x)\right|^{2} d x\right)^{1 / 2}$ In the below * means the transpose and the complex conjugate, and its integration is defined to be

$$
\int_{R^{4}} \lambda(x) d x=\left(\int_{R^{4}} \lambda_{1}(x) d x, \int_{R^{4}} \lambda_{2}(x) d x, \cdots \cdots, \int_{R^{4}} \lambda_{v}(x) d x\right)^{T} .
$$

The Fourier transform of $\lambda(x)$ is defined as $\hat{\lambda}(\gamma):=\int_{R^{4}} \lambda(x) \cdot e^{-i x \cdot \gamma} d x$, where $x \cdot \gamma$ denotes the inner product of real vectors $x$ and $\gamma$. For $\lambda, \hbar \in L^{2}\left(R^{4}\right)^{n}$, their symbol in ner product is defined by

$$
[\lambda(\cdot), \hbar(\cdot)]:=\int_{R^{4}} \lambda(x) \hbar(x)^{*} d x,
$$

Definition 1. We say that two vector functions $\lambda(x), \hbar(x) \in L^{2}\left(R^{4}\right)^{n}$ are biorthogonal ones, if

$$
[\lambda(\cdot), \hbar(\cdot-k)]=\delta_{\underline{0}, k} I_{n}, \quad k \in Z^{4},
$$

where $I_{n}$ denotes the $n \times n$ indentity matrix and $\delta_{\underline{0}, n}$ is the Kronecker symbol.

A sequence $\left\{\eta_{\imath}: \imath \in Z\right\} \subseteq \Omega$ is a frame for $\mathrm{H}$ if there exist positive real numbers $A, B$ such that

$$
\forall \lambda \in \Omega, \quad A\|\lambda\|^{2} \leq \sum_{i}\left|\left\langle\lambda, \eta_{t}\right\rangle\right|^{2} \leq B\|\lambda\|^{2}
$$

A sequence $\left\{\eta_{l}\right\} \subseteq \Omega$ is a Bessel sequence if (only) the upper inequality of(1) holds. If only for all $\lambda \in X \subset U$, the upper inequality of (1) holds, the sequence $\left\{\eta_{t}\right\} \subseteq \Omega$ is a Bessel sequence with respect to (w.r.t.) $\Omega$. If $\left\{\eta_{l}\right\}$ is a frame, there exist a dual frame $\left\{\eta_{l}^{*}\right\}$ such that

$$
\forall g \in \Omega, \quad g=\sum_{i}\left\langle g, \eta_{i}\right\rangle \eta_{i}^{*}=\sum_{i}\left\langle g, \eta_{i}^{*}\right\rangle \eta_{i}
$$

In what follows, we introduce the notion of vector- valued multiresolution analysis and give the definition of biorthogonal vector-valued wavelets of space $L^{2}\left(R^{4}\right)^{n}$.

Definition 3. A vector-valued multiresolution analysis of the space $L^{2}\left(R^{4}\right)^{n}$ is a nested sequence of closed subspaces $\left\{U_{v}\right\}_{v \in Z}$ such that (i) $U_{v} \subset U_{v+1}, \forall v \in Z$; (ii) $\bigcap_{v \in Z} U_{v}=\{0\}$ and $U_{v \in Z} U_{v}$ is dense in $L^{2}\left(R^{4}\right)^{n}$, where 0 denotes an zero vector of space $R^{v}$; (iii) $\hbar(x) \in U_{v} \Leftrightarrow \hbar(S x) \in U_{v+1}, \forall \mathrm{v} \in Z$; (iv) there exists $F(x) \in U_{0}$, called a vector-valued scaling function, such that its translates $F_{n}(x)$ : $=F(x-n), \quad n \in Z^{4}$ forms a Riesz basis of subspace $U_{0}$.

Since $F(x) \in Y_{0} \subset Y_{1}$, by Definition 3 and (4) there exists a finitely supported sequence of constant $v \times v$ matrice $\left\{P_{v}\right\}_{v \in Z^{4}} \in \ell^{2}\left(Z^{4}\right)^{v \times v}$ such that

$$
F(x)=\sum_{v \in Z^{4}} P_{v} F(S x-v) \text {. }
$$

Equation (6) is called a refinement equation. Define 


$$
\mathrm{P}(\gamma)=\frac{1}{a} \sum_{v \in Z^{4}} P_{v} \cdot \exp \{-i v \cdot \gamma\}, \quad \gamma \in R^{4}
$$

where $\mathbf{P}(\gamma)$, which is a $2 \pi Z^{4}$ periodic function, is called a symbol of $F(x)$. Thus, (6) becomes

$$
\hat{F}(S \gamma)=\mathrm{P}(\gamma) \hat{F}(\gamma), \quad \gamma \in R^{4} .
$$

Let $X_{j, j} \in Z$ be the direct complementary subspace of $U_{j}$ in $U_{j+1}$. Assume that there exist $a-1$ vector-valued functions $\Psi_{\mu}(x) \in L^{2}\left(R^{4}\right)^{n}, \mu \in \Gamma$ such that their tran- slations and dilations form a Riesz basis of $X_{j}$, i.e.,

$$
X_{j}=\overline{\left(\operatorname{span}\left\{\Psi_{\mu}\left(S^{j} \cdot-k\right): k \in Z^{4}, \mu \in \Gamma\right\}\right)}, \quad j \in Z .
$$

Since $\Psi_{\mu}(x) \in X_{0} \subset U_{1}, \quad \mu \in \Gamma$, there exist $a-1$ sequences of constant $v \times v$ matrice $\left\{B_{n}^{(\mu)}\right\}_{n \in Z^{4}}$ s.t.

$$
\Psi_{\mu}(x)=\sum_{v \in Z^{4}} B_{v}^{(\mu)} F(S x-v), \quad \mu \in \Gamma .
$$

By implementing the Fourier transform for the both sides of (9), we have

$$
\hat{\Psi}_{\mu}(4 \gamma)=\mathrm{B}^{(\mu)}(\gamma) \hat{\Phi}(\gamma), \quad \gamma \in R^{4}, \quad \mu \in \Gamma . \quad \mathrm{B}^{(\mu)}(\gamma)=\frac{1}{a} \sum_{v \in Z^{4}} B_{v}^{(\mu)} \cdot \exp \{-i v \cdot \gamma\}, \mu \in \Gamma .
$$

If $F(x), \tilde{F}(x) \in L^{2}\left(R^{4}\right)^{n}$ are a pair of biorthogonal vector-valued scaling functions, then

$$
[F(\cdot), \tilde{F}(\cdot-k)]=\delta_{\underline{0}, k} I_{n}, \quad k \in Z^{4} .
$$

We say that $\Psi_{\mu}(x), \tilde{\Psi}_{\mu}(x) \in L^{2}\left(R^{4}\right)^{n}, \mu \in \Gamma$ are pairs of biorthogonal vector-valued wavelets associated with a pairof biorthogonal vector-valued scaling functions $F(x)$ and $\tilde{F}(x)$, if the family $\left\{\Psi_{\mu}(x-n), n \in Z^{4}, \mu \in \Gamma\right\}$ Is a Riesz basis of subspace $X_{0}$, and

$$
\begin{aligned}
& {\left[F(\cdot), \tilde{\Psi}_{\mu}(\cdot-k)\right]=0, \quad \mu \in \Gamma, \quad k \in Z^{4} . \quad\left[\tilde{F}(\cdot), \Psi_{\mu}(\cdot-k)\right]=0, \quad \mu \in \Gamma, \quad k \in Z^{4} .} \\
& X_{j}^{(\mu)}=\overline{\operatorname{Span}\left\{\Psi_{\mu}\left(S^{j} \cdot-k\right): k \in Z^{4}\right\}}, \mu \in \Gamma, j \in Z .
\end{aligned}
$$

Similar to (5) and (9), there exist $a-1$ finite supported sequences of $v \times v$ constant matrice $\left\{\tilde{A}_{k}\right\}_{k \in Z^{4}}$ and $\left\{\tilde{B}_{k}^{(\mu)}\right\}_{k \in Z^{4}}, \mu \in \Gamma$ such that $\tilde{F}(x)$ and $\tilde{\Psi}_{\mu}(x)$ satisfy the refinement equations:

$$
\tilde{F}(x)=\sum_{k \in Z^{4}} \widetilde{P}_{k} \tilde{F}(S x-k), \quad \tilde{\Psi}_{\mu}(x)=\sum_{k \in Z^{4}} \tilde{B}_{k}^{(\mu)} \tilde{F}(S x-k), \mu \in \Gamma .
$$

\section{The biorthogonality propertiy of vector-valued wavelet packets}

Denoting by $H_{\underline{0}}(x)=F(x), \quad H_{\mu}(x)=\Psi_{\mu}(x), \widetilde{H}_{\underline{0}}(x)=\widetilde{F}(x), \quad \widetilde{H}_{\mu}(x)=\tilde{\Psi}_{\mu}(x), Q_{k}^{(0)}=P_{k}, Q_{k}^{(\mu)}=B_{k}^{(\mu)}$, $\tilde{Q}_{k}^{(0)}=\widetilde{P}_{k} \tilde{Q}_{k}^{(\mu)}=\tilde{B}_{k}^{(\mu)}, \mu \in \Gamma, k \in Z^{4}$. Let us order $S=4 I_{n}$. For any $\alpha \in Z_{+}^{4}$ and the given vector-valued biorthogonal scaling functions $G_{0}(x)$ and $\tilde{G}_{0}(x)$, iteratively define, respectively,

$$
H_{\alpha}(x)=H_{4 \sigma+\mu}(x)=\sum_{k \in Z^{4}} Q_{k}^{(\mu)} H_{\sigma}(4 x-k), \quad \widetilde{H}_{\alpha}(x)=\widetilde{H}_{4 \sigma+\mu}(x)=\sum_{k \in Z^{4}} \tilde{Q}_{k}^{(\mu)} \widetilde{H}_{\sigma}(4 x-k) .
$$

where $\mu \in \Gamma_{0}, \sigma \in Z_{+}^{4}$ is the unique element such that $\alpha=4 \sigma+\mu, \mu \in \Gamma_{0}$ follows.

Lemma $1^{[4]}$. Let $F(x), \tilde{F}(x) \in L^{2}\left(R^{4}, C^{v}\right)$. Then they are biorthogonal ones if and only if

$$
\sum_{k \in Z^{4}} \hat{F}(\gamma+2 k \pi) \hat{\tilde{F}}(\gamma+2 k \pi)^{*}=I_{v} .
$$


Definition 4. We say that two families of vector functions $\left\{H_{4 \sigma+\mu}(x), \sigma \in Z_{+}^{4}, \mu \in \Gamma_{0}\right\}$ and $\left\{\widetilde{H}_{4 \sigma+\mu}(x)\right.$, $\left.\sigma \in Z_{+}^{4}, \mu \in \Gamma_{0}\right\}$ are vector wavelet packets with respect to a pair of biorthogonal vector scaling functions $H_{\underline{0}}(x)$ and $\widetilde{H}_{\underline{0}}(x)$, respectively, where $H_{4 \sigma+\mu}(x)$ and $\widetilde{H}_{4 \sigma+\mu}(x)$ are given above.

Applying the Fourier transform for the both sides of (18) and (19) yields, respectively,

$$
\widehat{H}_{4 \sigma+\mu}(\gamma)=Q^{(\mu)}(\gamma / 4) \widehat{H}_{\sigma}(\gamma / 4), \quad \mu \in \Gamma_{0}, \quad \widehat{\widetilde{H}}_{4 \sigma+\mu}(4 \gamma)=Q^{(\mu)}(\gamma) \widehat{\widetilde{H}}_{\sigma}(\gamma), \quad \mu \in \Gamma_{0},
$$

Lemma $2^{[7]}$. Assume that $H_{\mu}(x), \widetilde{H}_{\mu}(x) \in L^{2}\left(R^{4}\right)^{n}, \mu \in \Gamma$ are pairs of biorthogonal vector-valued wavelets associated with a pair of biorthogonal scaling functions $H_{0}(x)$ and $\widetilde{H}_{\underline{0}}(x)$. Then, for $\mu, \lambda \in \Gamma_{0}$, we have $\sum_{\rho \vdash \Gamma^{*}} \mathrm{Q}^{(\mu)}((\gamma+2 \rho \pi) / 4) \tilde{Q}^{(\lambda)}((\gamma+2 \rho \pi) / 4)^{*}=\delta_{\mu, \lambda} I_{n}$.

Lemma $3^{[7]}$. Suppose ${ }^{\rho \in \Gamma_{0}}\left\{H_{\alpha}(x), \alpha \in Z_{+}^{4}\right\}$ and $\left\{\widetilde{H}_{\alpha}(x), \alpha \in Z_{+}^{4}\right\}$ are wavelet packets with respect to a pair of biorthogonal vector-valued functions $H_{\underline{0}}(x)$ and $\widetilde{H}_{\underline{0}}(x)$. Then, for $\alpha \in Z_{+}^{4}$, we have

$$
\left[H_{\alpha}(\cdot), \widetilde{H}_{\alpha}(\cdot-k)\right]=\delta_{\underline{0}, k} I_{v}, k \in Z^{4} .
$$

Theorem $\mathbf{1}^{[7]}$.Assume that $\left\{H_{\beta}(x), \beta \in Z_{+}^{4}\right\}$ and $\left\{\widetilde{H}_{\beta}(x), \beta \in Z_{+}^{4}\right\}$ are vector-valued wavelet packets with respect to a pair ofbiorthogonal vector-valued functions $H_{\underline{0}}(x)$ and $\widetilde{H}_{\underline{0}}(x)$, respectively. Then, for $\beta \in Z_{+}^{4}, \mu, v \in \Gamma_{0}$, we have

$$
\left[H_{4 \beta+\mu}(\cdot), \widetilde{H}_{4 \beta+v}(\cdot-k)\right]=\delta_{\underline{0}, k} \delta_{\mu, v} I_{v}, k \in Z^{4} .
$$

Theorem $2^{[7]}$. If $\left\{H_{\beta}(x), \beta \in Z_{+}^{4}\right\}$ and $\left\{\widetilde{H}_{\beta}(x), \beta \in Z_{+}^{4}\right\}$ are vector wavelet packets with respect to a pair of biorthogonal vector-valued scaling functions $H_{\underline{0}}(x)$ and $\widetilde{H}_{\underline{0}}(x)$, then for any $\alpha, \sigma \in Z_{+}^{4}$, we have

$$
\left[H_{\alpha}(\cdot), \widetilde{H}_{\sigma}(\cdot-k)\right]=\delta_{\alpha, \sigma} \delta_{\underline{0}, k} I_{v}, k \in Z^{4}
$$

\section{References}

[1] Q. Chen, etal, “A study on compactly supported orthogo-nal vector-valued wavelets and wavelet packets” . Chaos, Soli-tons \& Fractals. Vol.31, No.4, PP. 1024-1034,2007.

[2] G. Iovane, P. Giordano. "Wavelet and multiresolution analysis:Nature of $\varepsilon^{\infty}$ Cantorian space-time. Chaos", Solitons \& Frac-tals, Vol.32, No.4, PP.896-910,2007.

[3] N. Zhang, X.Wu X. "Lossless Compression of Color Mosaic Images” . IEEE Trans Image processing Vol. 15, No. 16, PP.1379-1388, 2006.

[4] Z. Shen," "Nontensor product wavelet packets in $L_{2}\left(R^{s}\right)$ ", SIAM Math. Anal., Vol. 26, No. 4,PP. 1061-1074, 1995.

[5] Q. Chen, Z. Cheng, X. Feng, "Multivariate Biorthogonal Multiwavelet packets," MATHEMATICA APPLICATA, in Chinese, 2005, 18(3), pp. 358-364.

[6] C. K. Chui, J. Lian, “A study of orthonormal multiwavelets”. Appli Numer Math, 1996, 20(1), 273-298.

[7] S. Yang. Z. Cheng H. Wang, "Construction of biorthogonal multiwavelets", Math Anal Appl, 2002, 276(1), 1-12. 